\title{
Temporal lobe structural evaluation after transsylvian selective amygdalohippocampectomy
}

\author{
Leonardo Giacomini, MD, ${ }^{1,3}$ Joao Paulo Sant Ana de Souza, MD, ${ }^{2}$ Cleiton Formentin, MD, ${ }^{1}$ \\ Brunno Machado de Campos, PhD, ${ }^{2}$ Alexandre B. Todeschini, MD, ${ }^{3}$ Evandro de Oliveira, MD, PhD, ${ }^{1}$ \\ Helder Tedeschi, MD, PhD, ${ }^{1}$ Andrei Fernandes Joaquim, MD, PhD, ${ }^{1}$ Fernando Cendes, MD, PhD, ${ }^{2}$ \\ and Enrico Ghizoni, MD, PhD'
}

Departments of ${ }^{1}$ Neurosurgery and ${ }^{2}$ Neurology, University of Campinas; and ${ }^{3}$ Department of Neurosurgery, Hospital 9 de Julho, São Paulo, Brazil

OBJECTIVE Mesial temporal lobe epilepsy (MTLE) is the most common type of focal epilepsy in adolescents and adults, and in $65 \%$ of cases, it is related to hippocampal sclerosis (HS). Selective surgical approaches to the treatment of MTLE have as their main goal resection of the amygdala and hippocampus with minimal damage to the neocortex, temporal stem, and optic radiations (ORs). The object of this study was to evaluate late postoperative imaging findings on the temporal lobe from a structural point of view.

METHODS The authors conducted a retrospective evaluation of all patients with refractory MTLE who had undergone transsylvian selective amygdalohippocampectomy (SAH) in the period from 2002 to 2015. A surgical group was compared to a control group (i.e., adults with refractory MTLE with an indication for surgical treatment of epilepsy but who did not undergo the surgical procedure). The inferior frontooccipital fasciculus (IFOF), uncinate fasciculus (UF), and ORs were evaluated on diffusion tensor imaging analysis. The temporal pole neocortex was evaluated using T2 relaxometry.

RESULTS For the IFOF and UF, there was a decrease in anisotropy, voxels, and fibers in the surgical group compared with those in the control group ( $p<0.001)$. An increase in relaxometry time in the surgical group compared to that in the control group ( $p$ 0.001) was documented, suggesting gliosis and neuronal loss in the temporal pole.

CONCLUSIONS SAH techniques do not seem to totally preserve the temporal stem or even spare the neocortex of the temporal pole. Therefore, although the transsylvian approaches have been considered to be anatomically selective, there is evidence that the temporal pole neocortex suffers structural damage and potentially functional damage with these approaches.

https://thejns.org/doi/abs/10.3171/2020.1.FOCUS19937

KEYWORDS temporal lobe epilepsy; epilepsy surgery; tractography; relaxometry

$\mathrm{M}$ ESIAL temporal lobe epilepsy (MTLE) is the most common type of focal epilepsy in adolescents and adults, and in $65 \%$ of cases, it is related to hippocampal sclerosis (HS). ${ }^{10,13}$ MTLE is typically seen in medically refractory epilepsy, and about $75 \%$ of these patients are resistant to medical management. ${ }^{27}$ Surgery has proven to be an effective treatment modality for patients with MTLE, with postoperative seizure-free rates ranging from $59 \%$ to $89 \% .^{11,29}$

Selective amygdalohippocampectomy (SAH) and an- terior temporal lobectomy (ATL) have produced similar postoperative seizure outcomes. ${ }^{3,24,25,28,35}$ However, some studies have indicated that SAH has better neuropsychological outcomes ${ }^{17,18}$ and a lower risk of visual field defects. ${ }^{23}$ On the other hand, some investigators have recorded neocortical epileptiform spikes with cortical electrodes, indicating that tailored resection of the temporal neocortex is essential for MTLE. ${ }^{2,26}$ Other studies have suggested that better seizure outcomes are achieved in patients who undergo ATL than in those who undergo $\mathrm{SAH},{ }^{14,15}$ and in

ABBREVIATIONS ATL = anterior temporal lobectomy; DTI = diffusion tensor imaging; FA = fractional anisotropy; HS = hippocampal sclerosis; IFOF = inferior frontooccipital fasciculus; MTLE = mesial temporal lobe epilepsy; OR = optic radiation; $\mathrm{SAH}=$ selective amygdalohippocampectomy; $\mathrm{TI}=$ transinsular; $\mathrm{TP}=$ temporal pole; $\mathrm{TS}=$ temporal stem; TU = transuncus; UF = uncinate fasciculus.

SUBMITTED November 30, 2019. ACCEPTED January 29, 2020.

INCLUDE WHEN CITING DOI: 10.3171/2020.1.FOCUS19937. 
still other studies, no significant differences in neuropsychological outcomes have been found between the surgical strategies. ${ }^{15,33}$

While some surgeons perform selective approaches to try to avoid injuries to the temporal neocortex, temporal stem (TS), and optic radiations (ORs), ${ }^{5,6}$ our group observed a high incidence of temporal pole (TP) atrophy on late postoperative MRI in patients who had undergone transsylvian SAH. ${ }^{14}$ This observation raises the question of whether transsylvian SAH is as selective as anatomically proposed. Therefore, in this paper, we aimed to study through advanced neuroimaging techniques any late postoperative effects to the temporal lobe and to understand the causes and consequences of TP atrophy.

\section{Methods \\ Study Design}

This is a retrospective nonrandomized study of all patients diagnosed with medically refractory MTLE surgically treated via transsylvian SAH compared to an ageand sex-matched control group of patients (i.e., adults with refractory MTLE with an indication for surgical treatment of epilepsy but who had not undergone the surgical procedure). The procedures were performed at the Epilepsy Center of the University of Campinas, Brazil, in the period from 2002 to 2015 . The study was approved by the ethics committee of the University of Campinas, and all patients provided written informed consent.

\section{Patient Population}

All patients had a diagnosis of unilateral MTLE with MRI evidence of HS and fulfilled the following criteria: a patient older than 18 years of age, clinical and electroencephalographic features of unilateral MTLE, standard preoperative investigation for epilepsy surgery, resection of the mesial structures of the temporal lobe via transsylvian $\mathrm{SAH}$, and late postoperative 3-T MRI (a minimum of 1 year after surgery).

The surgical group consisted of patients who had undergone surgical treatment via selective transsylvian techniques and were divided into two groups-either transinsular (TI) amygdalohippocampectomy or transuncus (TU) amygdalohippocampectomy. While some patients were potentially eligible for the surgical group (i.e., adults with refractory MTLE and an indication for surgical treatment of epilepsy), they had not undergone the procedure at the time of preoperative 3-T MRI. These patients made up the age- and sex-matched control group.

\section{Surgical Techniques}

In the period from 2002 to 2009, the transsylvian SAH was performed as described by Yaşargil et al., ${ }^{35}$ through the inferior circular sulcus of the insula, and we call this approach the "TI SAH" (Fig. 1A and B). Briefly, a sylvian fissure dissection is performed to expose the internal carotid artery, middle cerebral artery, uncus, limen insula, and inferior circular sulcus of the insula. The incision starts at the limen insula and progresses 1 to $2 \mathrm{~cm}$ posteriorly on the inferior circular sulcus to get to the temporal horn through its roof, that is, the TS. Once the temporal horn is reached, this incision is turned to the uncus, expos- ing the anterior portion of the hippocampus and temporal amygdala, and then the amygdalohippocampectomy is performed.

After 2009, we changed the TI approach to another transsylvian approach with an anteromedial route through the uncus to reach the temporal horn, which we called the "TU SAH" (Fig. 1C and D). ${ }^{14}$ The main proposal for, or change in, this approach was to reach the temporal horn while avoiding the TS and ORs. The approach was proposed by Coppens et al. ${ }^{9}$ and Choi et al. ${ }^{6}$ in elegant anatomical studies and has even been used by Yaşargil in his latest technical description. ${ }^{34}$

\section{Postoperative Advanced Imaging Evaluation}

The integrity of white matter fiber tracts was determined through 3-T MRI structural and diffusion tensor imaging (DTI) analysis of the most important fiber tracts related to temporal lobe surgery, namely the uncinate fasciculus (UF), inferior frontooccipital fasciculus (IFOF), and ORs (Fig. 2). ${ }^{20,30,31}$

Among the quantitative MRI techniques, T2 relaxometry is currently considered a very sensitive diagnostic tool for the identification of hippocampal abnormalities. ${ }^{4}$ Increased $\mathrm{T} 2$ relaxation time is related to tissue gliosis and ultimately neuronal loss. ${ }^{21} \mathrm{~T} 2$ relaxometry was used to evaluate the remaining TP about its integrity and to compare it with that in nonsurgical patients. As far as we know, this is the first study to use T2 relaxometry for structural assessment of the remaining temporal lobe after MTLE surgery. We defined the TP as the portion of the planum polare of the temporal lobe anterior to the limen insula.

\section{Diffusion Tensor Imaging}

DTI was performed using a single-shot multislice spin echo-planar TR of $8500 \mathrm{msec}$, TE of $60 \mathrm{msec}$, slice thickness of $2 \mathrm{~mm}$, matrix of $116 \times 115$, and FOV of 232 $\times$ 232. Initially, the DTI data sets and anatomical MRI scans were preprocessed for morphological suitability with SPM8 software (Wellcome Department of Cognitive Neurology, https://www.fil.ion.ucl.ac.uk), and maps were obtained with mean diffusivity values and fractional anisotropy (FA). From these maps, the calculation of DTI was performed with ExploreDTI software (A. Leemans, University Medical Center Utrecht).

The age- and sex-matched control group was used for comparing tract integrity. The integrity of the tracts was evaluated through FA, the number of voxels, and the number of fibers.

\section{Relaxometry}

For computation of the relaxometry values, images were acquired using multiecho $\mathrm{T} 2$ analysis with the following attributes: TR, $3300 \mathrm{msec}$; TE, 30/60/90/120/150 msec; slice thickness, $3 \mathrm{~mm}$; matrix, $200 \times 176$; and FOV, $1802 \times 180$. We used the Aftervoxel software (http://www.bergo.eng.br/ academic/aftervoxel) for quantification of the values. For each individual, single exponential decay equations were fit to the T2 imaging data obtained in each pixel, and then the $\mathrm{T} 2$ relaxation times were calculated for each pixel.

The region of interest in the T2-weighted images was semiautomatically defined in three consecutive coronal 


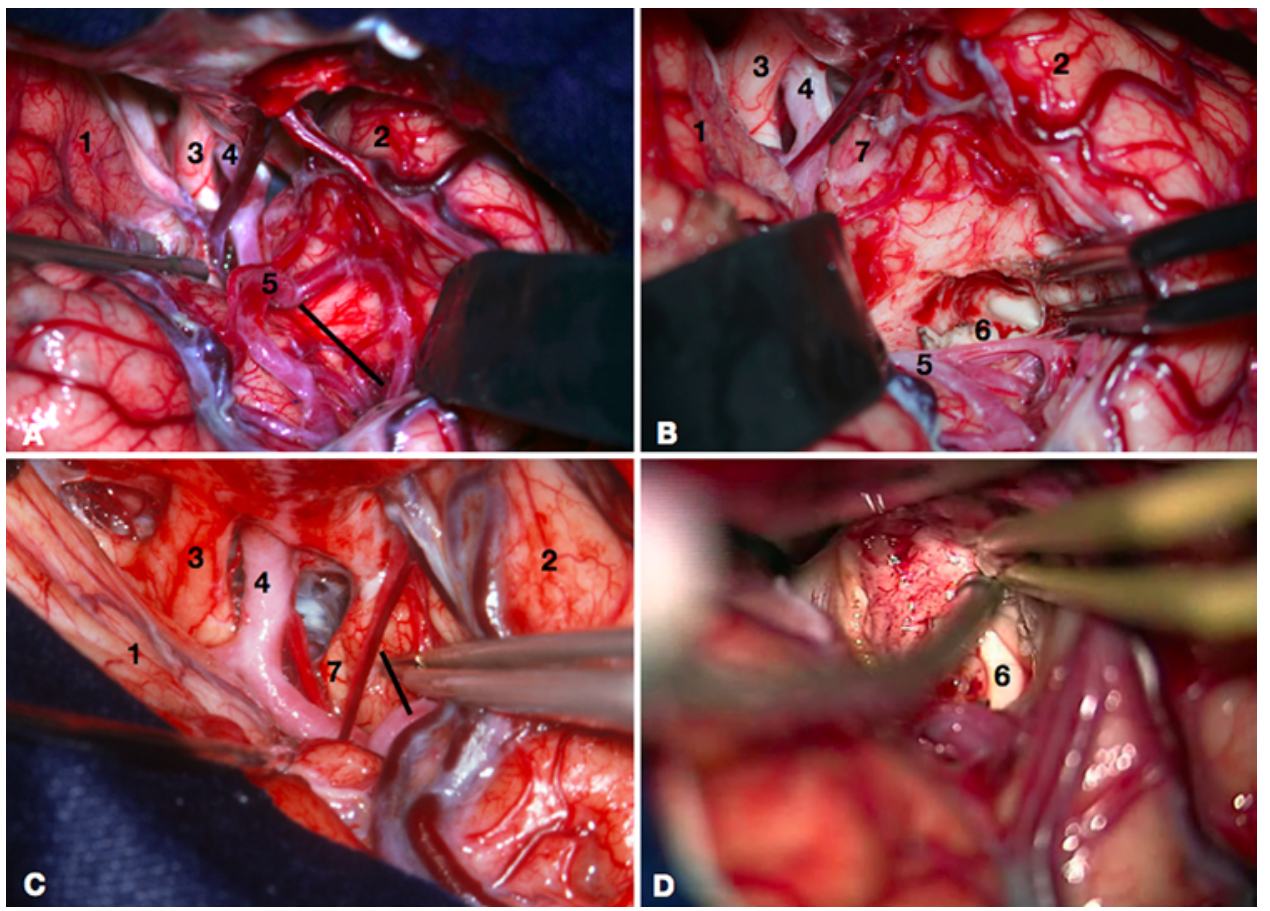

FIG. 1. A and B: Transinsular (TI) approach. The black line corresponds to the initial incision at the limen insula. C and D: Transuncus (TU) approach. The black line corresponds to the initial incision at the piriform cortex of the uncus. $1=$ frontal lobe; $2=$ temporal lobe; 3 = optic nerve; 4 = internal carotid artery; 5 = middle cerebral artery; 6 = temporal horn; 7 = uncus.

sections, using a $200 \%$ zoom and brush 7 , so that each region of interest was completely placed within the white matter of the TP. These parameters made it possible to distinguish gray matter from white matter better and to cover the larger area within the region of interest. The mean T2 signal of the three sections of each TP was used as the final measure.

The three regions of interest of the patients who had undergone surgical treatment were placed in the same sections of the control group to obtain comparisons between corresponding analog areas. The regions of interest were placed in the first coronal section in which the distinction between gray and white matter was completely visible to the naked eye at the two TPs (operated and unoperated sides). Blind analysis of the data was impossible since the surgically treated temporal lobe had clear signal abnormality in most patients.

\section{Statistical Analysis}

The variables were presented in terms of their values of central tendency and dispersion. The Levene and Kolmogorov-Smirnov tests were performed to verify homogeneity of the variances and adherence to the normal curve, respectively. To compare the three groups (TI SAH, TU SAH, and control), the Kruskal-Wallis test was used, followed by Dunn's multiple comparison test. The level of significance was $5 \%$.

\section{Results}

\section{Demographic Data}

We evaluated 86 patients divided into three groups. The surgical group consisted of 56 patients who had undergone either TI SAH (29 patients) or TU SAH (27 patients). The control group comprised 30 adult MTLE patients. The demographic data for each group were as follows: TI $\mathrm{SAH}$, mean age $46.38 \pm 8.01$ years (mean \pm standard deviation), 15 females, 14 males; TU SAH, mean age 45.11 \pm 9.2 years, 18 females, 9 males; and control, mean age $41.17 \pm 11.50$ years, 18 females, 12 males. Patients in each surgical group were matched by sex and age with patients in the control group (Table 1).

\section{Inferior Frontooccipital Fasciculus}

There were interruptions in IFOF fibers in $50 \%$ of the patients who had undergone SAH (23/29 in TI group and $5 / 27$ in TU group). In the control group, all patients had preserved IFOF. It was observed by the Kruskal-Wallis test that the FA variable differs among the three treatment groups, and by Dunn's multiple comparison test, we found that the TI group differs from the TU group $(\mathrm{p}<0.005)$

TABLE 1. Demographic data according to treatment group: surgical (TI and TU) and control

\begin{tabular}{cccc}
\hline Variable & No. of Males & No. of Females & Mean Age $(\mathrm{yrs})$ \\
\hline Group & & & \\
\hline TI SAH & 14 & 15 & $46.38 \pm 8.01$ \\
\hline TU SAH & 9 & 18 & $45.11 \pm 9.2$ \\
\hline Control & 12 & 18 & $41.17 \pm 11.50$ \\
\hline p value & $>0.05$ & $>0.05$ & $>0.05$ \\
\hline
\end{tabular}



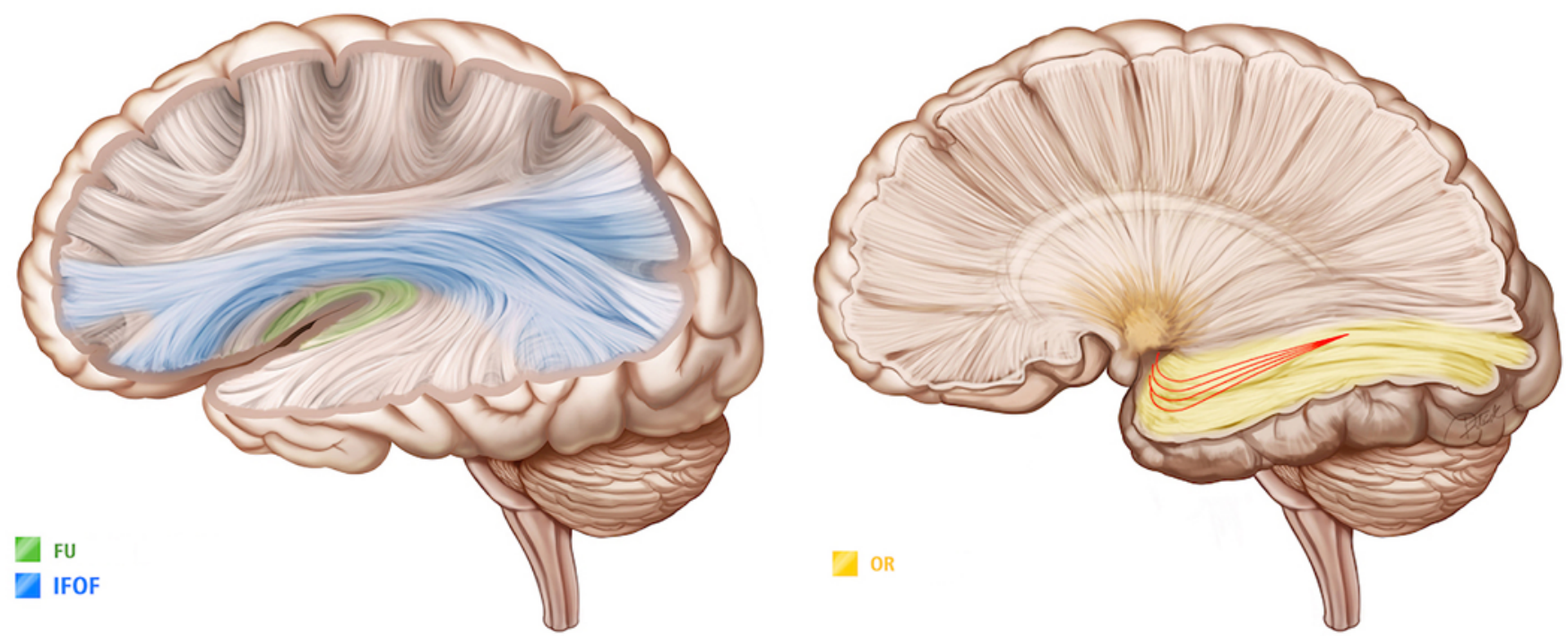

FIG. 2. IFOF (blue), UF (green), OR (yellow), and Meyer's loop (red). Copyright MedPixel. Published with permission.

and that the two SAH groups differ from the control group $(p<0.005)$. For the voxels variable, it was observed by the Kruskal-Wallis test that the groups are different, and by Dunn's multiple comparison test, we found that the TI group differs from the TU group $(\mathrm{p}<0.005)$ and that the two SAH groups differ from the control group $(\mathrm{p}<0.005)$. For the variable FA, it was observed by the Kruskal-Wallis test that the groups are different, and by Dunn's pairwise comparisons, we found differences between the TI and TU groups $(\mathrm{p}<0.005)$ and between the control group and the SAH groups ( $<<0.005$; Fig. 3 ).

\section{Uncinate Fasciculus}

The UF evaluated in the 56 patients who had undergone SAH via either technique was interrupted on tractography in $94.64 \%$ of them (29/29 in TI group and 24/27 in TU group). In the control group, all the patients presented with preserved tractography of the UF.

The Kruskal-Wallis test showed that the FA variable differs among the three groups, and per Dunn's multiple comparison test, the TI group differs from the TU group $(\mathrm{p}<0.005)$ and the two SAH groups differ from the control group $(\mathrm{p}<0.005)$. For the voxels variable, it was observed by the Kruskal-Wallis test that the groups are different, but according to Dunn's multiple comparison test, the TI group does not differ from the TU group $(p>0.05)$. However, the two SAH groups differ from the control group: TI differs from control $(\mathrm{p} \leq 0.05)$ and TU differs from control ( $\mathrm{p} \leq 0.05)$. For the variable number of fibers, it was observed by the Kruskal-Wallis test that the groups are different, and per Dunn's multiple comparison test, the TI group does not differ from the TU group $(\mathrm{p}>0.05)$, although the two groups differ from the control group $(\mathrm{p}$ $<0.005$; TI group differs from control group, $\mathrm{p} \leq 0.05$; TU group differs from control group, $\mathrm{p} \leq 0.05$; Fig. 4).

\section{Optic Radiations}

Analysis of the tractography of the ORs showed inter- ruption of the fibers in $8.92 \%$ of the patients, with interruption only in the TI group (5/29) and preservation of the fibers in all patients in the TU and control groups.

It was observed by the Kruskal-Wallis test that the FA variable does not differ among the three groups $(p=0.45)$. For the voxels variable, it was observed by the KruskalWallis test that the groups are different, and by Dunn's multiple comparison test, the TI group differs from the TU group $(\mathrm{p}<0.005)$ and from the control group $(\mathrm{p}<$ $0.005)$. The TU group does not differ from the control group ( $p>0.05)$. For the variable fibers, it was observed by the Kruskal-Wallis test that the groups are different, but it was not possible to detect in which group the differences were attributable to the great dispersion of the TI group (Fig. 5).

\section{Relaxometry}

Three regions of the temporal lobe white matter were defined in patients who had undergone TI SAH (29 patients) and TU SAH (27 patients) and in members of the control group (30 patients). There was a difference in relaxometry values among the groups $(\mathrm{p} \leq 0.05$, KruskalWallis test), and Dunn's pairwise comparisons showed differences between TI and controls $(p<0.005)$ and between TU and controls ( $\mathrm{p}<0.005)$, but there was no difference between TI and TU groups (Fig. 6).

\section{Discussion}

Yaşargil et al. described the transsylvian SAH, proposing to remove the amygdala and hippocampus through a ventricular route sparing the temporal neocortex, which is different from the ATL and the selective transcortical approaches. ${ }^{35}$ The SAH became one of the most popular approaches to treat MTLE resistant to medical management among centers specializing in epilepsy surgery. However, with the rebirth of studies on the white matter substance of the brain, for example by Türe et al., ${ }^{30}$ and the use of DTI tractography studies, great interest in the 


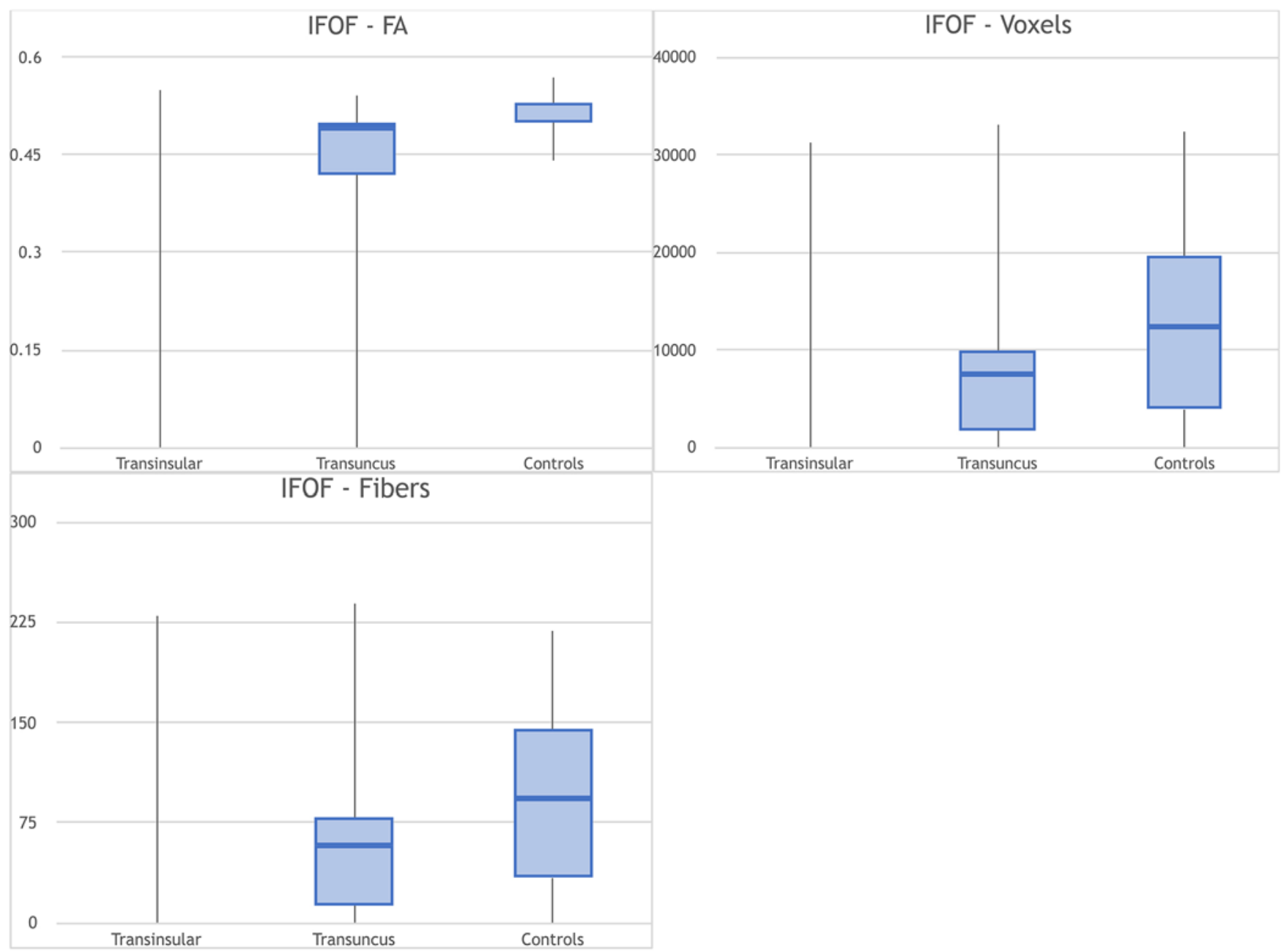

FIG. 3. Analysis by study group for IFOF, according to FA (y-axis represents degree of anisotropy), number of voxels (y-axis), and number of fibers (y-axis).

anatomy of the white matter fiber tracts resurged. The TS is a bridge of white matter connecting the temporal lobe to the basal ganglia, where several important fiber tracts (UF, IFOF, and ORs) extend anteriorly from the amygdala, following the inferior circular sulcus of the insula. ${ }^{22} \mathrm{Sev}-$ eral authors have noticed that the initial description of the SAH technique by Yaşargil et al. included an incision of 1-2 $\mathrm{mm}$ behind the limen insula in the inferior circular sulcus of the insula to gain access to the temporal horn. ${ }^{34}$ That means that the temporal horn of the lateral ventricle was approached through a structure called the "temporal stem." In elegant anatomical studies, Coppens et al. and Choi et al. proposed an anteromedial route through the anterior segment of the uncus to reach the temporal horn, trying to avoid the TS. ${ }^{6,9}$ The main proposal for, or change in, this approach is to reach the temporal horn while avoiding the TS and ORs. In his most recent publication about epilepsy surgery, Yaşargil describes the approach through the amygdala, diverging from his initial technical description. ${ }^{34}$ Following this anatomical paradigm change and trying to validate this new anatomical proposition for an anteromedial approach through the uncus to reach the temporal horn, we started a prospective analysis of a series of patients who underwent TU SAH. ${ }^{14}$ The objective was to check if the TU approach was more effective in protecting the TS and its contents than the traditional TI approach. However, our results showed a high incidence of postoperative TP atrophy $(69.6 \%$ in the TU approach and $100 \%$ in the TI approach); therefore, we decided to analyze with advanced neuroimaging techniques the remaining TP.

To evaluate the remaining TP tissue, we used T2 relaxometry, which, by increasing the relaxation time, identifies the presence of brain gliosis, which ultimately represents neuronal loss. ${ }^{12}$ Analysis of the increased relaxation time on T2-weighted images was initially used as a tool to increase the sensitivity in detecting HS, especially in patients with few or no abnormalities on MRI studies. ${ }^{4,8}$ Bernasconi et al. used T2 relaxometry as an indication for amygdalohippocampectomy in patients with MTLE (clinical and electroencephalographic diagnosis) with MRI studies without evidence of HS. Increased relaxation time in conjunction with data provided by electroencephalography helps in the correct lateralization of the 
Giacomini et al.

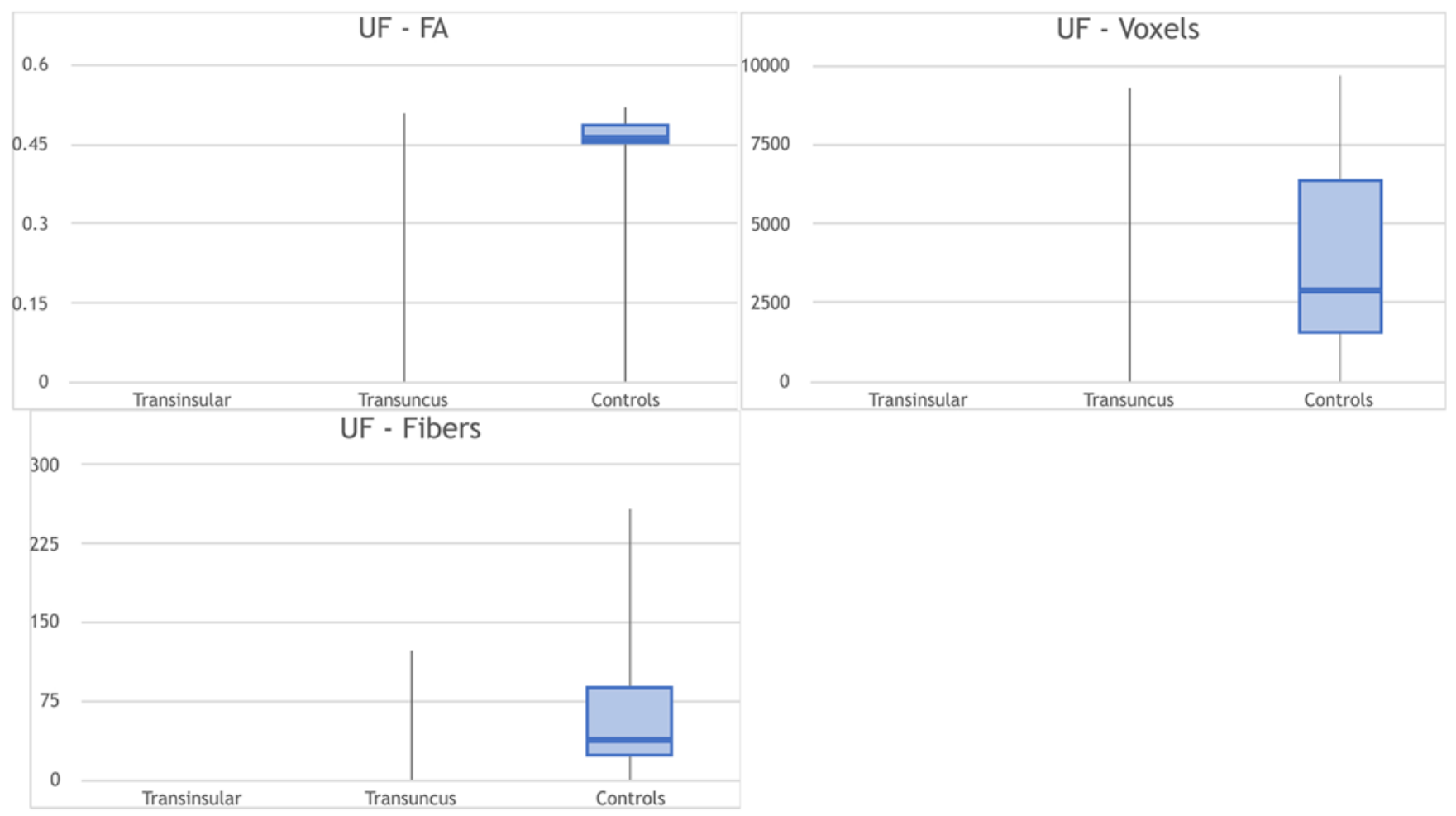

FIG. 4. Analysis by study group for UF, according to FA (y-axis represents degree of anisotropy), number of voxels (y-axis), and number of fibers (y-axis).

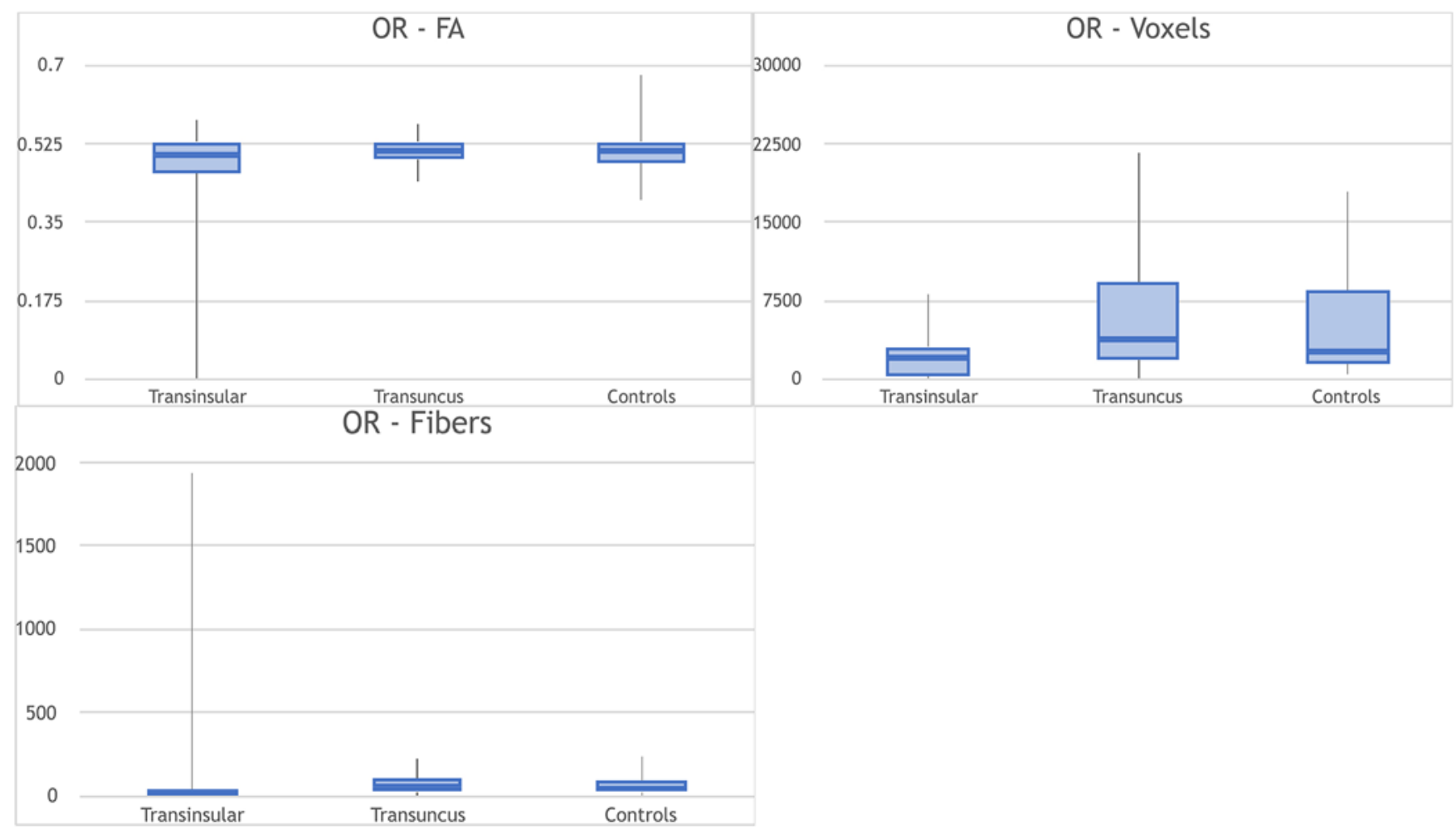

FIG. 5. Analysis by study group for ORs, according to FA (y-axis represents degree of anisotropy), number of voxels (y-axis), and number of fibers (y-axis). 


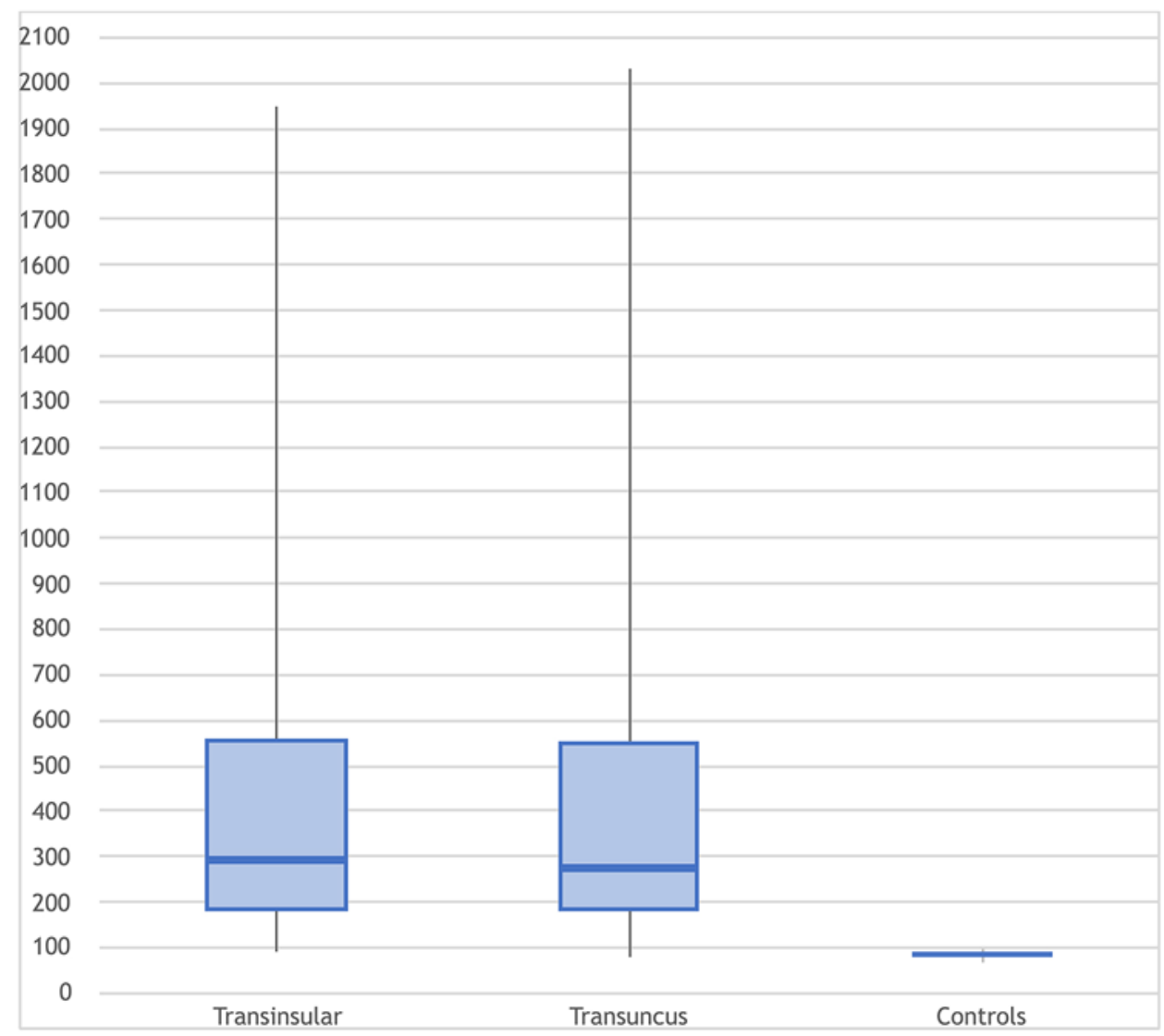

FIG. 6. Analysis of relaxometry by study group. The y-axis represents the relaxation times in milliseconds.

epileptogenic focus, confirmed by the marked presence of gliosis in immunohistochemical studies. ${ }^{4}$ Coan et al. demonstrated a $28 \%$ increase in HS identification after the inclusion of $\mathrm{T} 2$ relaxometry and hippocampal volumetric evaluation in MTLE patients evaluated at an epilepsy center. ${ }^{8}$ Our results showed an unquestionable increase in the relaxation time in the remaining TP in patients undergoing SAH, both TI and TU, compared to that in the control group, suggesting that despite anatomical preservation of the temporal neocortex, there was severe neuronal loss and the tissue became gliotic with questionable function. Indeed, gliotic tissue is a recognized pathology related to epilepsy and can be related to late surgery failure. ${ }^{14}$ Therefore, if one assumes that after selective approaches, some gliotic tissue remains in the temporal lobe, these remnants can be used to explain the results of the Josephson et al. meta-analysis, which showed the superiority of ATL over selective approaches to treat MTLE. ${ }^{19}$

Our DTI results showed the superiority of the TU approach over the TI approach to preserve the ORs and the IFOF, but both approaches showed a high incidence of UF interruption (TU: $88.9 \%$; TI: 100\%). When analyzing the two groups together (SAH group) and separately (TU and TI), a clear statistical difference ( $\mathrm{p} \leq 0.05)$ with the control group in the three evaluated parameters was found, showing evident lesioning of this tract after surgical treatment. The insular segment of the UF is found in the limen insula, with an average width of $7 \mathrm{~mm}$ and a height of 5 $\mathrm{mm},{ }^{32}$ and given that the incision of the TI approach starts at the limen insula, it is clear that is the cause of UF interruption in all patients. Regarding the TU approach, we hypothesize that it involves a narrow surgical field for the resection of deeply seated mesial structures and requires more retraction and manipulation of the anterior portion of the TS, which can increase the risk of brain injury and tissue ischemia. ${ }^{14}$ Another important tract is the IFOF, which travels in a more superficial layer than the ORs. ${ }^{14}$ It presented an interruption rate of $79.31 \%$ (23/29) in patients undergoing the TI approach and $18.51 \%(5 / 27)$ in patients undergoing the TU approach, but the IFOF in these cases had a decrease in voxels and fibers compared to those in the control group, suggesting some damage.

About the causes of TP atrophy, we can assume that they must be related to UF damage (UF connects the orbitofrontal cortex and frontal pole with the TP) and to the inherent disconnection from the uncus, amygdala, hippocampus, and parahippocampal gyrus promoted by amygdalohippocampectomy. In addition, studies have suggested that the remaining TP in patients undergoing SAH may play a significant role in promoting epileptiform activity from the TP neocortex. ${ }^{1}$ Abel et al. reported the results of a study on intraoperative TP electrocorticography in 10 consecutive patients who had undergone SAH with the previous diagnosis of HS without any change in the TP on neuroimaging studies. ${ }^{1}$ Of the 10 patients evaluated, 7 had neocortical epileptiform peaks from the TP, suggest- 
ing its role in promoting seizures in these patients. That study serves to question the real importance of preserving the TP in selective techniques.

Extraventricular approaches, such as subtemporal (collateral sulcus) or supracerebellar transtentorial SAH, can potentially minimize risk to the UF, IFOF, and ORs, at least from an anatomical point of view. ${ }^{7,16,18}$ We encourage other epilepsy centers with their different selective approaches to review their postoperative images to search for TP atrophy and additional white matter tract injuries. The results of different techniques have the potential to clarify if the postoperative atrophy is attributable to TS white matter damage or if it is a consequence of the disconnection of the hippocampus and amygdala from the neocortex

It is worth highlighting some limitations of this study, including its nature as a retrospective, nonrandomized, single-center study and its lack of postoperative neuropsychological data. The aim of this study was to conduct a structural analysis of the remaining temporal lobe and the possible reasons for postoperative TP atrophy. The white matter findings of this study will be used in future studies to evaluate their functional consequences comparing them to the neuropsychological outcomes and connectivity findings of the remaining TP.

Selective techniques demand more technical skills and more technological resources (neuronavigation, ultrasonic aspirator) and do not provide adequate specimens (body of the hippocampus) for research purposes. If other centers confirm our results with different selective techniques, ATL could become the standard of treatment for refractory MTLE patients.

\section{Conclusions}

The increase in the $\mathrm{T} 2$ relaxation time of the remaining TP in patients who had undergone SAH via TI and TU techniques revealed the presence of gliosis and neuronal loss, thus indicating structural impairment of the remaining anterior temporal neocortex. There was also significant structural injury of the FU and IFOF in both of the techniques considered to be selective (TU and TI approaches) and of the ORs in the TI approach. Together these data suggest that despite the use of selective surgical techniques, anterior temporal lobe neocortex suffers some structural damage and possibly functional damage as well.

\section{References}

1. Abel TJ, Woodroffe RW, Nourski KV, Moritani T, Capizzano AA, Kirby P, et al: Role of the temporal pole in temporal lobe epilepsy seizure networks: an intracranial electrode investigation. J Neurosurg 129:165-173, 2018

2. Alarcon G, Garcia Seoane JJ, Binnie CD, Martin Miguel MC, Juler J, Polkey CE, et al: Origin and propagation of interictal discharges in the acute electrocorticogram. Implications for pathophysiology and surgical treatment of temporal lobe epilepsy. Brain 120:2259-2282, 1997

3. Bate H, Eldridge P, Varma T, Wieshmann UC: The seizure outcome after amygdalohippocampectomy and temporal lobectomy. Eur J Neurol 14:90-94, 2007

4. Bernasconi A, Bernasconi N, Caramanos Z, Reutens DC, Andermann F, Dubeau F, et al: T2 relaxometry can lateralize mesial temporal lobe epilepsy in patients with normal MRI. Neuroimage 12:739-746, 2000

5. Campero A, Trócoli G, Martins C: Fernandez-Miranda JC, Yasuda A, Rhoton AL Jr: Microsurgical approaches to the medial temporal region: an anatomical study. Neurosurgery 59 (4 Suppl 2):ONS279-ONS308, 2006

6. Choi C, Rubino PA, Fernandez-Miranda JC, Abe H, Rhoton AL Jr: Meyer's loop and the optic radiations in the transsylvian approach to the mediobasal temporal lobe. Neurosurgery 59 (4 Suppl 2):ONS228-ONS236, 2006

7. Clusmann H, Kral T, Gleissner U, Sassen R, Urbach H, Blümcke I, et al: Analysis of different types of resection for pediatric patients with temporal lobe epilepsy. Neurosurgery 54:847-860, 2004

8. Coan AC, Kubota B, Bergo FP, Campos BM, Cendes F: 3T MRI quantification of hippocampal volume and signal in mesial temporal lobe epilepsy improves detection of hippocampal sclerosis. AJNR Am J Neuroradiol 35:77-83, 2014

9. Coppens JR, Mahaney KB, Abdulrauf SI: An anteromedial approach to the temporal horn to avoid injury to the optic radiation fibers and uncinate fasciculus: anatomical and technical note. Neurosurg Focus 18(6B):E3, 2005

10. Crawford PM: Epidemiology of intractable focal epilepsy, in: Oxbury JM, Polkey CE, Duchowny M: Intractable Focal Epilepsy, ed 1. London: WB Saunders, 2000, pp 25-52

11. Falconer MA, Taylor DC: Surgical treatment of drug-resistant epilepsy due to mesial temporal sclerosis. Etiology and significance. Arch Neurol 19:353-361, 1968

12. Fawcett JW, Asher RA: The glial scar and central nervous system repair. Brain Res Bull 49:377-391, 1999

13. Foldvary N: Symptomatic focal epilepsies, in Wyllie E (ed): The Treatment of Epilepsy, ed 3. Philadelphia: Lippincott Williams \& Wilkins, 2001, pp 467-474

14. Ghizoni E, Matias RN, Lieber S, de Campos BM, Yasuda CL, de Souza JPSAS, et al: Clinical and imaging evaluation of transuncus selective amygdalohippocampectomy. World Neurosurg 100:665-674, 2017

15. Goldstein LH, Polkey CE: Behavioural memory after temporal lobectomy or amygdalo-hippocampectomy. Br J Clin Psychol 31:75-81, 1992

16. Harput MV, Türe U: The paramedian supracerebellartranstentorial selective amygdalohippocampectomy for mediobasal temporal epilepsy. Oper Neurosurg (Hagerstown) 14:462, 2018

17. Helmstaedter C, Reuber M, Elger CC: Interaction of cognitive aging and memory deficits related to epilepsy surgery. Ann Neurol 52:89-94, 2002

18. Hori T, Yamane F, Takenobu A: Microanatomy of medial temporal area and subtemporal amygdalohippocampectomy. Stereotact Funct Neurosurg 77:208-212, 2001

19. Josephson CB, Dykeman J, Fiest KM, Liu X, Sadler RM, Jette N, et al: Systematic review and meta-analysis of standard vs selective temporal lobe epilepsy surgery. Neurology 80:1669-1676, 2013

20. Kier EL, Staib LH, Davis LM, Bronen RA: MR imaging of the temporal stem: anatomic dissection tractography of the uncinate fasciculus, and Meyer's loop of the optic radiation. AJNR Am J Neuroradiol 25:677-691, 2004

21. Marshall VG, Bradley WG Jr, Marshall CE, Bhoopat T, Rhodes RH: Deep white matter infarction: correlation of MR imaging and histopathologic findings. Radiology 167:517522, 1988

22. Martino J, Vergani F, Robles SG, Duffau H: New insights into the anatomic dissection of the temporal stem with special emphasis on the inferior fronto-occipital fasciculus: implications in surgical approach to left mesiotemporal and temporoinsular structures. Neurosurgery 66 (3 Suppl Operative): $4-12,2010$

23. Mengesha T, Abu-Ata M, Haas KF, Lavin PJ, Sun DA, Kon- 
rad PE, et al: Visual field defects after selective amygdalohippocampectomy and standard temporal lobectomy. J Neuroophthalmol 29:208-213, 2009

24. Niemeyer P: The transventricular amygdala-hippocampectomy in the temporal lobe epilepsy, in Baldwin M, Bailey P (eds): Temporal Lobe Epilepsy. Springfield, IL: Charles C Thomas, 1958, pp 461-482

25. Paglioli E, Palmini A, Portuguez M, Paglioli E, Azambuja N, da Costa JC, et al: Seizure and memory outcome following temporal lobe surgery: selective compared with nonselective approaches for hippocampal sclerosis. J Neurosurg 104:7078, 2006

26. Schwartz TH, Bazil CW, Walczak TS, Chan S, Pedley TA, Goodman RR: The predictive value of intraoperative electrocorticography in resections for limbic epilepsy associated with mesial temporal sclerosis. Neurosurgery 40:302-311, 1997

27. Spencer SS, Berg AT, Vickrey BG, Sperling MR, Bazil CW, Shinnar S, et al: Predicting long-term seizure outcome after resective epilepsy surgery: the multicenter study. Neurology 65:912-918, 2005

28. Tanriverdi T, Olivier A, Poulin N, Andermann F, Dubeau F: Long-term seizure outcome after mesial temporal lobe epilepsy surgery: cortical amygdalohippocampectomy versus selective amygdalohippocampectomy. J Neurosurg 108:517524, 2008

29. Téllez-Zenteno JF, Dhar R, Wiebe S: Long-term seizure outcomes following epilepsy surgery: a systematic review and meta-analysis. Brain 128:1188-1198, 2005

30. Türe U, Yaşargil MG, Friedman AH, Al-Mefty O: Fiber dissection technique: lateral aspect of the brain. Neurosurgery 47:417-427, 2000

31. Wang F, Sun T, Li XG, Liu NJ: Diffusion tensor tractography of the temporal stem on the inferior limiting sulcus. J Neurosurg 108:775-781, 2008

32. Wiebe S, Blume WT, Girvin JP, Eliasziw M: A randomized, controlled trial of surgery for temporal-lobe epilepsy. N Engl J Med 345:311-318, 2001
33. Wolf RL, Ivnik RJ, Hirschorn KA, Sharbrough FW, Cascino GD, Marsh WR: Neurocognitive efficiency following left temporal lobectomy: standard versus limited resection. J Neurosurg 79:76-83, 1993

34. Yaşargil MG, Krayenbühl N, Roth P, Hsu SP, Yaşargil DC: The selective amygdalohippocampectomy for intractable temporal limbic seizures. J Neurosurg 112:168-185, 2010

35. Yaşargil MG, Teddy PJ, Roth P: Selective amygdalo-hippocampectomy. Operative anatomy and surgical technique. Adv Tech Stand Neurosurg 12:93-123, 1985

\section{Disclosures}

The authors report no conflict of interest concerning the materials or methods used in this study or the findings specified in this paper.

\section{Author Contributions}

Conception and design: Giacomini. Acquisition of data: Giacomini, de Souza, de Campos. Analysis and interpretation of data: Giacomini, de Souza, Formentin, de Campos, Ghizoni. Drafting the article: Giacomini, Formentin, Todeschini, Joaquim, Ghizoni. Critically revising the article: Tedeschi, Joaquim, Cendes, Ghizoni. Reviewed submitted version of manuscript: Giacomini, de Souza, Formentin, Todeschini, de Oliveira,

Tedeschi, Joaquim, Cendes, Ghizoni. Approved the final version of the manuscript on behalf of all authors: Giacomini. Statistical analysis: de Souza, de Campos. Administrative/technical/material support: de Campos, de Oliveira, Tedeschi, Cendes, Ghizoni. Study supervision: Cendes, Ghizoni.

\section{Correspondence}

Leonardo Giacomini: University of Campinas, São Paulo, Brazil. giacominineurocirurgia@gmail.com. 